\title{
Managing Informal Mathematical Knowledge: Techniques from Informal Logic
}

\author{
Andrew Aberdein \\ Humanities and Communication, \\ Florida Institute of Technology, \\ 150 West University Blvd, \\ Melbourne, Florida 32901-6975, U.S.A. \\ aberdein@fit.edu
}

\begin{abstract}
Much work in MKM depends on the application of formal logic to mathematics. However, much mathematical knowledge is informal. Luckily, formal logic only represents one tradition in logic, specifically the modeling of inference in terms of logical form. Many inferences cannot be captured in this manner. The study of such inferences is still within the domain of logic, and is sometimes called informal logic. This paper explores some of the benefits informal logic may have for the management of informal mathematical knowledge.
\end{abstract}

\section{Informal Mathematical Knowledge}

What sort of mathematical knowledge does mathematical knowledge management manage? A distinction between knowledge that and knowledge how is frequently deployed in epistemology. In mathematics this corresponds to the distinction between knowing mathematical propositions and knowing how to conduct mathematical proofs, that is being acquainted with mathematical practice. Each of these two sorts of knowledge may be related to a problem for MKM. In the first case, the problem may be expressed as 'How can a computer represent the truths of mathematics?'. In recent years this problem has been tackled with increasing success. In the second case, the problem may be expressed as 'How can a computer represent the proofs of mathematics?'. If this question is understood as 'How can a computer perform the proofs of mathematics?', then the progress in automated theorem proving provides a ready answer. However, this would be to misunderstand the original question, which did not ask how mathematics could be done by a machine, but how it is and has been done by mathematicians.

The traditions of formalization and automated theorem proving upon which much work in MKM has been based are heavily indebted to the methods of formal logic. However, formal logic is a poor guide to mathematical practice, as mathematicians seldom use it to write proofs. Although most mathematical proofs may in principle be formalized, the process is often arduous and can dramatically reduce intelligibility. For this reason such formalization is rarely 
attempted, and most mathematicians regard formal logic as of little relevance to their work. Moreover, a great deal of important mathematical communication does not even aspire to be formalizable in principle. Why? Because it contains substantial gaps, or even contradictions.

At first glance, it may seem that such mathematics would be no great loss. However, there are several areas of mathematical practice to which it is indispensable. The first of these is the history of mathematics. Little if any mathematical work conducted before the twentieth century meets modern standards of rigour. An historian engaged in a diachronic study of a mathematical theory needs to marshal a considerable body of mathematical inference, much of it unsound. Salvaging the sound parts and restating them with modern rigour may be good practice for textbook writers, but is not acceptable for historians.

Contemporary mathematics can also give rise to similar problems. The refereed journal article is not the only form of mathematical communication. Mathematicians with shared interests can often communicate complex ideas with considerable brevity and absence of formal rigour. Moreover, collaborators often profit from sharing their work in an unpolished, and perhaps mistaken, form. The famous English mathematicians G. H. Hardy and J. E. Littlewood apparently ran their long-standing and successful collaboration in accordance with a set of 'axioms'. The first of these 'said that when one wrote to the other (they often preferred to exchange thoughts in writing instead of orally), it was completely indifferent whether what they said was right or wrong. As Hardy put it, otherwise they could not write completely as they pleased, but would have to feel a certain responsibility thereby.' (from a lecture by Harald Bohr, cited in Littlewood, 1986, p. 10).

That sort of communication may be inadvertently contradictory, but some mathematicians have gone further, to find heuristic insight in 'the idea ... that a proof can be respectable without being flawless' (Lakatos, 1976, p. 139). Consider, for example, the following remarks of Fields medallist Vaughan Jones:

I used to dislike intensely, but have come to appreciate and even search for ... the situation where one has two, watertight well-designed arguments that lead inexorably to opposite conclusions. ... Remember that research in mathematics involves a foray into the unknown. We may not know which of the two conclusions is correct or even have any feeling or guess. Proof at this point is our only arbiter. And it seems to have let us down. I have known myself to be in this situation for months on end. It induces obsessive and anti-social behaviour. Perhaps we have found an inconsistency in mathematics. But no, eventually a crack is found in one of the arguments and it begins to look more and more shaky. Eventually we kick ourselves for being so utterly stupid and life goes on. But it was no tool of logic that saved us. The search for a chink in the armour often involved many tricks including elaborate thought experiments and perhaps computer calculations. Much structural understanding is created, which is why I now so value this process. One's feeling of having obtained truth at the end is approaching the absolute. Though I should add that 
I have been forced to reverse the conclusion on occasions (Jones, 1998, pp. 208 f., emphasis added).

The situation which Jones describes, a deeper mathematical understanding derived from the analysis of apparently rebutted proofs, is central to the mathematical methodology espoused by the Hungarian philosopher of mathematics Imre Lakatos. His 'method of proofs and refutations' was inspired by the attempts of several generations of mathematicians to rescue the Descartes-Euler Conjecture from numerous apparent rebuttals. He summarizes the method as follows:

Rule 1. If you have a conjecture, set out to prove it and to refute it. Inspect the proof carefully to prepare a list of non-trivial lemmas (proof-analysis); find counterexamples both to the conjecture (global counterexamples) and to the suspect lemmas (local counterexamples).

Rule 2. If you have a global counterexample discard your conjecture, add to your proof-analysis a suitable lemma that will be refuted by the counterexample, and replace the discarded conjecture by an improved one that incorporates the lemma as a condition. Do not allow a refutation to be dismissed as a monster. Try to make all 'hidden lemmas' explicit.

Rule 3. If you have a local counterexample, check to see whether it is also a global counterexample. If it is you can easily apply Rule 2 . (Lakatos, 1976, p. 50).

Rule 4. If you have a counterexample which is local but not global, try to improve your proof analysis by replacing the refuted lemma by an unfalsified one. (Lakatos, 1976, p. 58).

Rule 5. If you have counterexamples of any type, try to find, by deductive guessing, a deeper theorem to which they are counterexamples no longer. (Lakatos, 1976, p. 76).

The mathematician described by Lakatos's method accrues mathematical beliefs, but many of them are tentative, changeable, and quite possibly wrong. But if Lakatos is right, and Jones's experience suggests that he is, this is nevertheless one of the most successful strategies for acquiring mathematical knowledge.

\section{Informal Logic}

\subsection{What is Informal Logic?}

To speak of informal logic is not to contradict oneself but to acknowledge what should be obvious: that the understanding of natural arguments requires substantive knowledge and insights not captured in the axiomatized rules of formal logic. (Govier, 1987, p. 204). 
The distinction between formal and informal logic has been stated in a variety of different ways. Much of the confusion arises from the ambiguity of 'formal' (Johnson, 1996, p. 45). Informal logic does not exclude the pursuit of precise and normative principles by which arguments may be analyzed and evaluated. Rather it concerns itself with arguments which cannot reliably be represented purely in terms of the logical form of the component propositions. Characteristically, these are arguments expressed in natural language. However, as the last section suggests, some arguments in mathematics may also have this quality. ${ }^{1}$

\subsection{The Toulmin Layout}

One of the most influential attempts to analyze the structure of arguments without appealing to the logical form of their propositions was developed in the 1950s by Stephen Toulmin. His 'layout' can represent deductive inference, but encompasses many other species of argument besides. The arguments it analyzes may vary considerably in strength: in particular, they may be defeasible - Toulmin was one of the first philosophers to use this term. Toulmin's layout continues to be an important focus for contemporary work in informal logic.

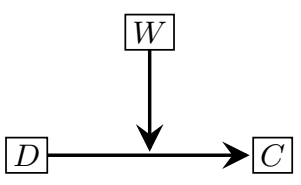

(a) Basic Layout

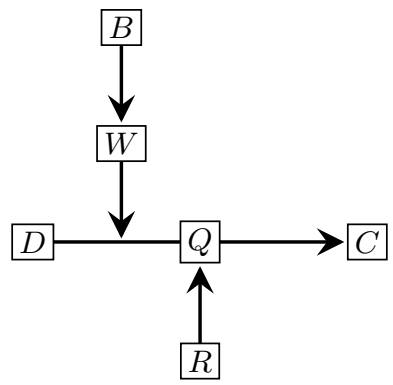

(b) Enhanced Layout

Fig. 1. Toulmin layouts

In its simplest form, shown in Fig. 1(a), the layout represents the derivation of a Claim $(C)$, from Data $(D)$, in accordance with a Warrant $(W)$. This DWC pattern may appear to resemble a deductive inference rule, such as modus ponens, but it can be used to represent many other, looser inferential steps. The differences between these types of inference are made explicit by the additional elements of the full layout shown in Fig. 1(b). The warrant is justified by its dependence on Backing $(B)$, possible exceptions or Rebuttals $(R)$ are allowed

\footnotetext{
${ }^{1}$ For a more substantial defence of the applicability of informal logic to mathematics,
} see Aberdein (2006). 
for, and the resultant force of the argument is stated in the Qualifier $(Q)$. Hence the full layout may be understood as 'Given that $D$, we can $Q$ claim that $C$, since $W$ (on account of $B$ ), unless $R$ '. For example: 'Given that Harry was born in Bermuda, we can presumably claim that he is British, since anyone born in Bermuda will generally be British (on account of various statutes ...), unless his parents were aliens, say'. ${ }^{2}$
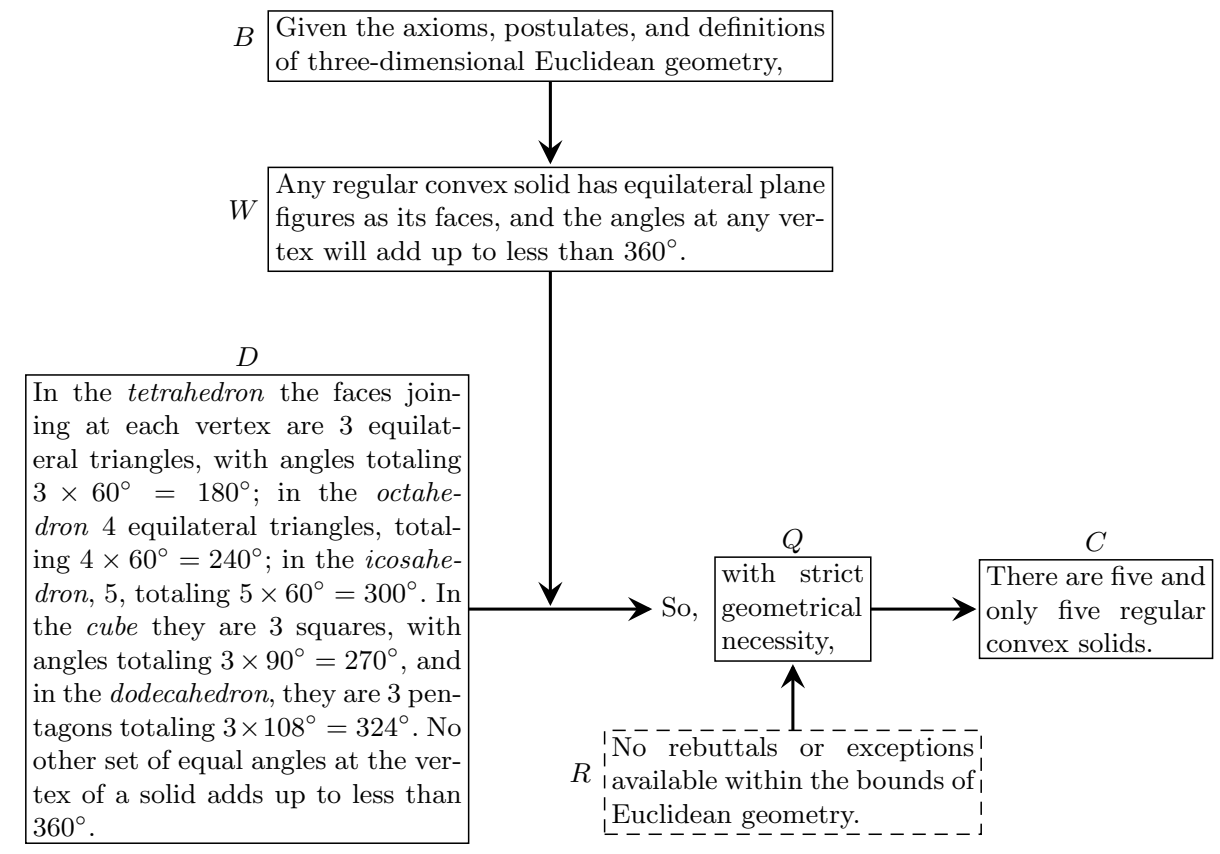

Fig. 2. Toulmin's analysis of Theaetetus's proof that the platonic solids are exactly five in number (adapted from Toulmin \& al., 1979, Fig. 7.4, p. 89).

Toulmin's focus is on argumentation in natural language, not mathematics, although he is satisfied that the layout applies there as well. However, his only developed mathematical example is the proof from Euclid reproduced here as Fig. 2. One substantial shortcoming that this proof has as a model for how Toulmin's layout may be applied more generally is that it has only one step. Most mathematical proofs have many. Moreover, the number of steps a proof possesses is a function of the detail with which it is presented. In the next section I develop an extension of Toulmin's system which permits the presentation of multi-step proofs and also exhibits the relationship between presentations of

$\overline{2}$ A frequently used example, derived from Toulmin (1958, p. 104). 
differing depths of detail. Before doing so, I shall pause briefly to discuss an idiosyncrasy of Toulmin's approach: its highly visual nature.

\subsection{The Pros and Cons of Visual Presentation}

There are clear benefits to be gained from the visual presentation of argument, as a growing body of research acknowledges (see Kirschner \& al., 2003, for example). A shared visual presentation can significantly facilitate communication of complex ideas, whether collaborative or pedagogic. The Toulmin layout, which is usually represented graphically, is a good example of this. However, visual presentation also has its drawbacks. Diagrams can be time-consuming to produce and frustrating to update, comment upon or integrate with other systems. As the diagrams grow in complexity these problems escalate.

At least two responses may be made to these difficulties. Firstly, these problems can largely be eliminated through the use of suitable software. Several programs are now available which, to a greater or lesser extent, automate the process of argument diagraming. Some of these programs (such as Araucaria: see Reed \& Rowe, 2005) can be adapted to represent Toulmin layouts. Secondly, the convention of representing Toulmin layouts diagrammatically aids understanding, but is not essential. The basic layout may be thought of as a triple, $\langle D, W, C\rangle$. The greater generality of the enhanced layout may be brought to bear by associating a further triple $\langle B, Q, R\rangle$ with the warrant. We can therefore represent a full Toulmin layout as $\langle D, W\langle B, Q, R\rangle, C\rangle$, where each component represents a set of propositions, except for $Q$, a single term.

\section{Combining Basic Layouts}

\subsection{Four Principles for Combining Layouts}

Various proposals have been made to extend Toulmin's layout (for example, Newman \& Marshall, 1992, pp. 15 f.). Many of these are concerned with applications of the layout to types of argument unlikely to occur in mathematical proof. I propose the following principles for combining mathematical layouts:

I Treat data and claim as the nodes in a graph or network.

II Allow nodes to contain multiple propositions.

III Any node may function as the data or claim of a new layout.

IV The whole network may be treated as data in a new layout.

Principle I: I shall not consider ways of extending the layout by adding links to components other than the data or claim. This often seems to duplicate existing features of the layout. For example, making the warrant of one layout the claim of a second duplicates the role of backing, albeit with more structure. Where such extensions are original, as in Newman \& Marshall's (1992, p. 24) attachment of data and warrant to a rebuttal, they frequently seem more appropriate for other contexts, such as the legal argumentation for which this extension was devised. 
Further to the argument in Sect. 2.3, we may observe that although graphs are frequently set out diagrammatically, this is not essential. Strictly speaking, a directed graph comprises a finite set of vertices, or nodes, and a finite multiset of edges, or ordered pairs of vertices. Each simple layout within a compound layout will correspond to such an ordered pair. Hence, in a network of layouts $\left\langle D_{i}, W_{i}\left\langle B_{i}, Q_{i}, R_{i}\right\rangle, C_{i}\right\rangle, D_{i}$ and $C_{i}$ label the vertices, and $W_{i}$ and the other components label the edges.

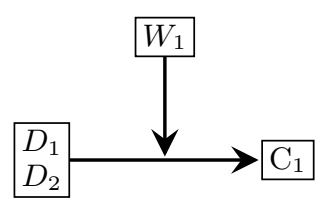

(a) Linked

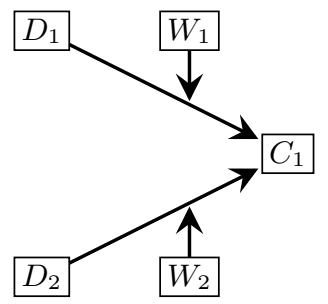

(c) Convergent

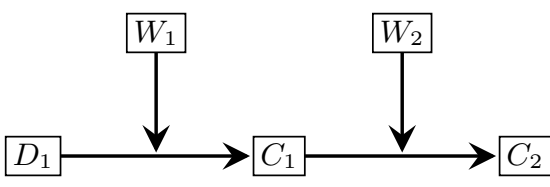

(b) Sequential

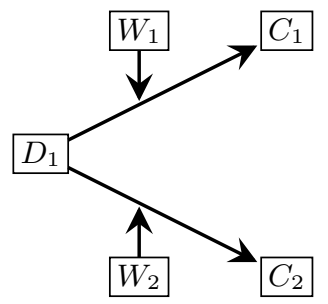

(d) Divergent

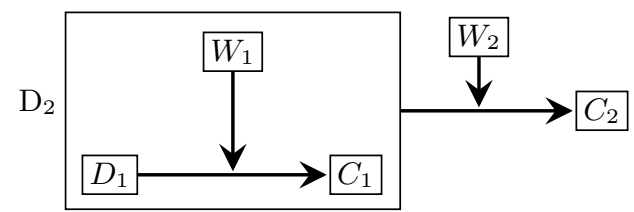

(e) Embedded

Fig. 3. Five ways of combining layouts.

Principle II: Toulmin already permits multiple data: consider the data in Fig. 2. This allows him to capture the linked argument structure represented as Fig. 3(a). We shall go beyond Toulmin in permitting multiple propositions within a node to be distinguished as separate nodes (represented graphically by nested boxes). However, this is unnecessary unless the propositions are individually attached to other nodes. 
In practice, we will still treat claims as singular. There may be some economy of exposition to be gained in permitting multiple claims to function as implicit disjunctions, in the manner of multiple conclusion sequent calculi. However, since nodes may function as both data and claim, this would risk unnecessary confusion.

Principle III: This principle allows us to construct sequential, convergent and divergent arguments, represented in Fig. 3(b)-(d). Sequential layouts are briefly considered in Toulmin \& al. (1979, p. 79); convergent and divergent arguments do not seem to be addressed. Strict adherence to Principle III prevents the occurrence of circuits, that is nodes which may be reached by two separate paths. (So the graphs we produce are actually trees.) Circuits are inferentially benign, provided they are acyclic - that is non-question-begging. However, they represent a redundancy of derivation which is seldom found in mathematical proofs. We could modify the principle to include acyclic circuits, but at the cost of complicating the folding rules introduced in Section 3.2.

Principle IV: This is our most radical departure from Toulmin, but we shall see that it is essential in order to capture some of the most pervasive forms of mathematical argument. Indeed, the reification of proofs as objects within larger proofs was a fundamental step in the development of mathematics. It is rather less common in ordinary discourse, making this situation dual to those we set aside in discussion of Principle I.

Principle IV does not allow embedding of a network into the claim of a new layout. The effect of such a step would be that the data and warrant of the new layout justified the derivation contained in the embedded network, placing them more naturally within the backing of (the individual steps of) the latter network. Principle III does permit data containing an embedded network to be treated as the claim of a new layout. However, in practice we shall avoid this move, restricting embedding to initial data.

Figure 4 exhibits how these principles may be used to reproduce some of the most common techniques in mathematical proof. Adjunction, Fig. 4(a), is just an instance of the linked layout, Fig. 3(a). Proof by contradiction, Fig. 4(b), combines divergent, Fig. 3(d), and embedded, Fig. 3(e), layouts. Representing each leg of the divergent layout separately would produce a logically equivalent presentation of this argument as a combination of linked and embedded layouts. This is the combination employed in both proof by cases, Fig. 4(c), and induction, Fig. 4(d).

\subsection{Folding Compound Layouts into a Single Layout}

Is it possible to 'fold' the steps of a compound layout into a single layout? This process may obscure much of the detail of a proof, but should preserve soundness. That is, the folded proof should be no less sound than the unfolded proof: depending on its qualifiers, this may not itself be sound. 


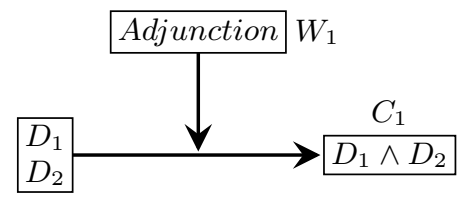

(a) Adjunction

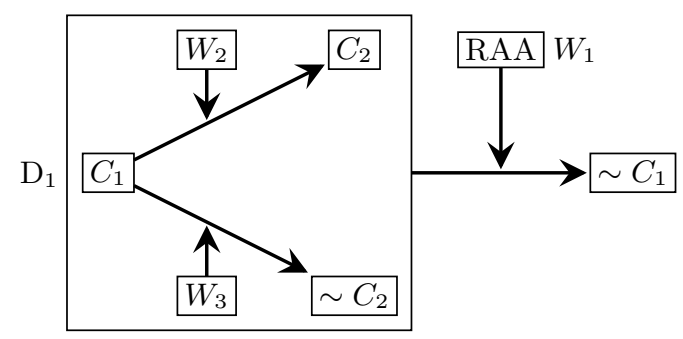

(b) Proof by Contradiction

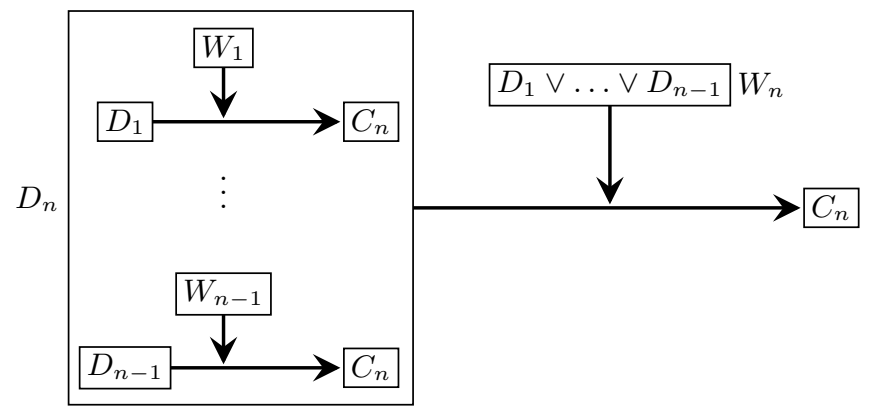

(c) Proof by Cases

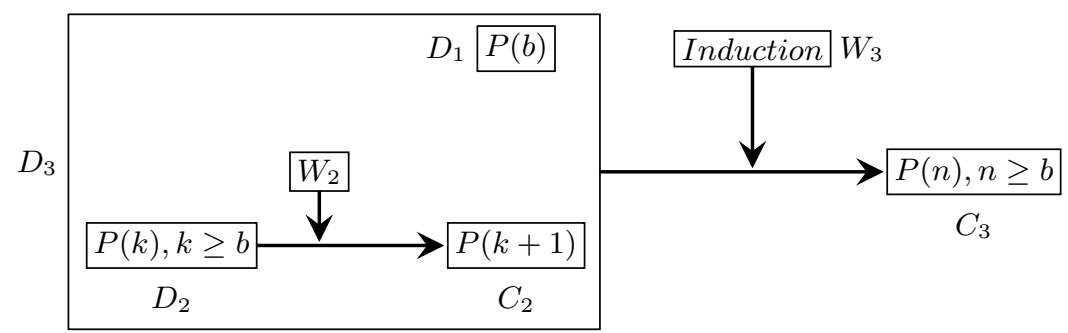

(d) (Weak) Induction

Fig. 4. Some common proof methods. 
To see how this may be done, we will first observe that any network satisfying Principles I-IV must have at least one node of in-degree zero, which is not derived from anything, and at least one node of out-degree zero, from which nothing is derived. We shall call the former nodes initial, the latter final, and all other nodes intermediate. The folded layout should exhibit the dependency of the final nodes on the initial nodes; the intermediate nodes may be ignored. To preserve soundness, the warrant of the folded layout must be sufficient to justify each step of the unfolded proof. For networks following Principles I-III only, that is without embedding, a folded layout which meets these requirements may be defined as follows:

$$
\left\langle\bigcup_{\operatorname{in}\left(D_{i}\right)=0} D_{i}, \bigwedge_{i} W_{i}, \bigwedge_{\operatorname{out}\left(C_{i}\right)=0} C_{i}\right\rangle
$$

The warrant, $W$, of the folded layout is thus defined as the conjunction of every warrant, $W_{i}$, in the unfolded layout. This guarantees the inferential resources necessary to carry out the proof, although in practice a more concise warrant may suffice. The minimal requirement is that $W \Rightarrow W_{i}$ for all $W_{i}$, where ' $\Rightarrow$ ' represents an appropriate account of derivation, which at this point we are assuming is used indifferently throughout the proof.

To deal with Principle IV, start with the most deeply embedded network(s) and reduce each to a simple layout, as in (1). There may be multiple layouts embedded in the same box, as in Fig. 4(c): see the next section for a worked example. Then replace each embedded layout with a new data node $D_{j}^{\#}$ defined as $D_{j} \Rightarrow C_{j}$, where $D_{j}$ and $C_{j}$ are the data and claim of the embedded layout(s). Finally, conjoin the warrant(s), $W_{j}$, of each embedded layout to that of the layout in which it was embedded, $W_{k}$. The following simple layout will result:

$$
\left\langle\bigcup_{j}\left(D_{j} \Rightarrow C_{j}\right), W_{k} \wedge \bigwedge_{j} W_{j}, C_{k}\right\rangle
$$

In representing the embedded layout propositionally, we are again assuming that a single account of derivation is in use throughout the proof.

By applying these rules recursively, it is possible to reduce a compound layout of arbitrary complexity to a simple layout. Of course, such a reduction will omit much detail, but it will preserve soundness. As a simple example, consider the representation of proof by contradiction in Fig. 4(b). Applying (1) to the embedded argument produces a simple layout $\left\langle C_{1}, W_{2} \wedge W_{3}, C_{2} \wedge \sim C_{2}\right\rangle$. We may then use (2) to produce a single layout representing the whole argument, $\left\langle C_{1} \Rightarrow\left(C_{2} \wedge \sim C_{2}\right), W_{1} \wedge W_{2} \wedge W_{3}, \sim C_{1}\right\rangle$.

\subsection{An Extended Example}

Figure 5 shows how the techniques introduced above may be applied to a real example, in this case the proof that every natural number greater than one has 


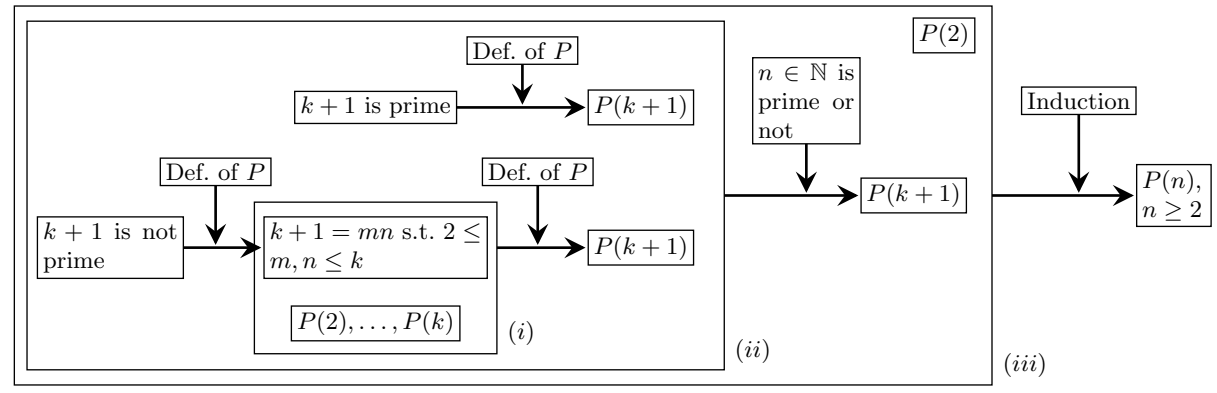

Fig. 5. Proof that every natural number greater than one has a prime factorization. $(P(n)$ abbreviates ' $n$ has a prime factorization'.)

a prime factorization. The proof is by induction, in this case strong induction, rather than the weak induction exhibited in Fig. 4(d), since all the preceding cases are included in the antecedent of the inductive step. Moreover, the inductive step is itself a proof by cases, so we have more than one level of embedding. I have indicated the nested boxes in the network as $(i)-(i i i)$. However, box $(i)$ is not a case of embedding, but rather a sequential pair of layouts in which new data is added at the intermediate stage. This network, and the simple layout above it are the two cases which constitute the proof of the inductive step. As such they are embedded in the data of that step, here indicated as (ii). Together with the base step, $P(2)$, the inductive step is itself embedded in the data (iii) of the outermost layout.

By applying the folding rules from Section 3.2, we may fold the whole proof into a single layout,

$$
\begin{aligned}
&\langle\{P(2),\{k+1 \text { is prime } \Rightarrow P(k+1), \\
&\{k+1 \text { is not prime, } P(2), \ldots, P(k)\} \Rightarrow P(k+1)\}\Rightarrow P(k+1)\}, \\
& \text { Def. of } P \wedge n \in \mathbb{N} \text { is prime or not } \wedge \text { Induction, } \\
&P(n) \text { for } n \geq 2\rangle .
\end{aligned}
$$

This just says that the desired result follows from the base case and the inductive step, in accordance with induction and the warrants for the inductive step. Clearly, if the unfolded proof is sound, the folded proof must be too.

\section{Enhanced Layouts}

For the sake of simplicity I have so far only considered combinations of layouts which lack backing, qualifiers or rebuttals. It is reasonable to omit these from analyses of the steps of a proof when they are identical in every step. This is true of many mathematical proofs. However, in some of the most interesting cases it is not. The folding rules from Sect. 3.2 may be augmented to include these 
additional components as follows:

$$
\begin{gathered}
\left\langle\bigcup_{\operatorname{in}\left(D_{i}\right)=0} D_{i}, \bigwedge_{i} W_{i}\left\langle\bigwedge_{i} B_{i}, \operatorname{lub} \bigcup_{i} Q_{i}, \bigvee_{i} R_{i}\right\rangle, \bigwedge_{\operatorname{out}\left(C_{i}\right)=0} C_{i}\right\rangle \\
\left\langle\bigcup_{j}\left(D_{j} \Rightarrow Q_{Q_{j}} C_{j}\right), W_{k} \wedge \bigwedge_{j} W_{j}\left\langle B_{k} \wedge \bigwedge_{j} B_{j}, \operatorname{lub}\left\{Q_{k} \cup \bigcup_{j} Q_{j}\right\}, R_{k} \vee \bigvee_{j} R_{j}\right\rangle, C_{k}\right\rangle
\end{gathered}
$$

These rules are conservative of those for the more primitive components, with one exception. Once we permit differently qualified steps in the same proof we may no longer assume that all derivations have equal force. The univocal concept of derivation used in (2) is therefore indexed to the prevailing qualifier in (5).

The justification of the rules for backing and rebuttals is straightforward. We treat backing identically to warrants, by conjoining all the individual backings to ensure a common backing sufficient for the folded layout. Since the rebuttal of a single step is enough to rebut the entire proof, the compound rebuttal is just the disjunction of all the individual rebuttals. Combining qualifiers takes a little more care. To do so we need to make some preliminary assumptions. Firstly, that all qualifiers occurring more than once in a derivation are non-cumulative. We shall describe a qualifier $Q$ as non-cumulative iff it holds of a compound layout of $n$ steps, each with qualifier $Q$ itself. Typically qualifiers indicating dependence on some axiom or assumption are non-cumulative, whereas qualifiers indicating likelihood are cumulative. However, for small values of $n$, qualifiers indicating high, but not absolute, levels of confidence may be treated as non-cumulative, since multiplying the possibility of error by the number of steps would still yield a very low number. ${ }^{3}$ Secondly, we must assume that the different qualifiers $Q_{i}$ may be given a partial ordering, such that $Q_{j} \leq Q_{k}$ iff every $Q_{j}$-qualified step is a $Q_{k}$-qualified step. For example, every constructively valid step is also classically valid, so 'constructively' $\leq$ 'classically'. Finally, we shall assume that every pair of qualifiers has a least upper bound (lub). Note that this qualifier need not itself be attached to any step of the proof.

The qualifier of the compound layout may then be defined as the least upper bound of the qualifiers of the individual steps. In some pathological cases the different steps of a proof may appeal to mutually inconsistent standards of rigour, for instance classical and Brouwerian intuitionistic mathematics. Here the least upper bound of the two qualifiers will be something falling far short of mathematical rigour (of either kind), such as 'perhaps', since the proof must be invalid.

The use of qualifiers (and backing) in a layout can make explicit the different assumptions and standards of rigour underlying different steps of the proof. We can see this in the classically but not constructively valid proof of the Intermediate Value Theorem laid out in Fig. 6. This proof has several steps which are constructively (and therefore also classically) valid, here folded together into

\footnotetext{
${ }^{3}$ A number representing an upper bound on the possibility of error of the whole layout, since the sources of error associated with each step need not be independent.
} 


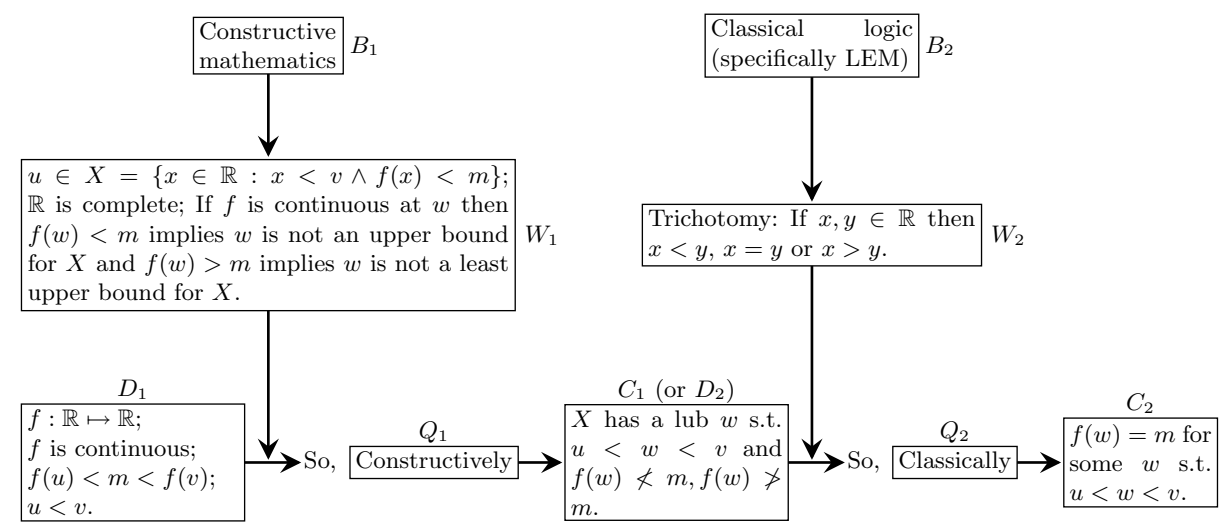

Fig. 6. Classical proof of the Intermediate Value Theorem.

the first step. However, there is at least one step with the qualifier 'classically', hence 'classically' must also be the compound qualifier, since it is the least upper bound of 'constructively' and 'classically'.

\section{$5 \quad$ Rebuttals}

The system developed above is a potentially powerful tool for the rational reconstruction of mathematical proofs. It bears some similarity to projects such as Leslie Lamport's 'structured proofs' (1995) or Michael Kohlhase's OMDoc (2000). In particular, the derive steps which comprise the OMDoc proof environment correspond fairly closely to basic Toulmin layouts. However, these steps lack any analogue to either the qualifier or the rebuttal component of a full layout. In this respect OMDoc (and structured proofs) are fit to their primary purpose: to facilitate greater formalization of proofs. Conversely, my approach is primarily intended to respect the level of (in)formality with which the proof was originally framed. Central to this pursuit is the rebuttal component. In this final section I explore how it may enrich our understanding of mathematical proof.

Ostensibly, Toulmin denies that mathematical arguments can ever be rebutted: observe the place holder for rebuttal in Fig. 2. He does accept that mathematical arguments are open to criticism, but only by challenging their 'standards of rational adequacy' (Toulmin \& al., 1979, p. 133). Individual proofs may be undermined by wide-ranging shifts in mathematical rigour, but they are not subject to more specific rebuttal. But this is just to say that Toulmin's focus is on formal, not informal, mathematics. His is an entirely reasonable attitude to take to settled and formalized mathematical results. However, in the context of informal mathematics, it is equally reasonable to admit the possibility of rebuttal. 


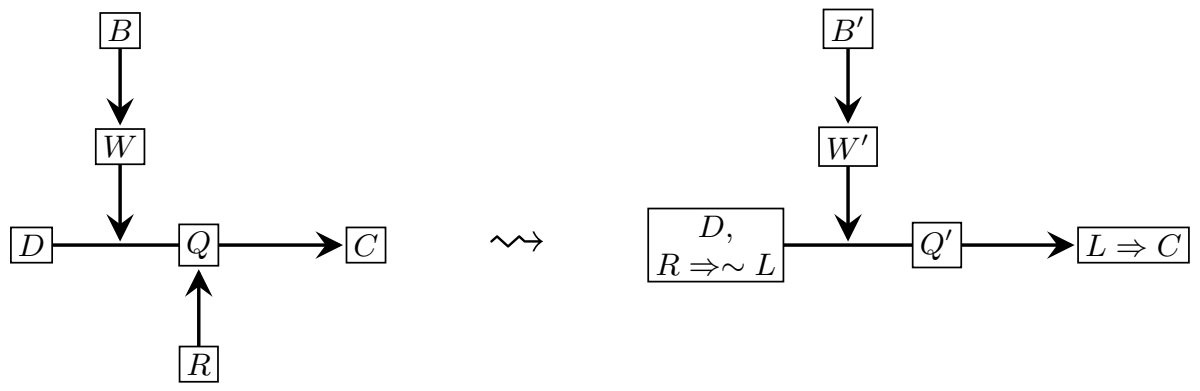

Fig. 7. Lemma incorporation

The ease with which Lakatos's rules lend themselves to translation into the idiom developed in this paper helps to confirm its usefulness in the analysis of informal mathematics. In the first rule, the conjecture corresponds to the (final) claim of a network of Toulmin layouts representing the proof. The lemmas are the initial data, the global counterexamples rebuttals of the final step in the proof, and the local counterexamples rebuttals to earlier steps. The second rule, Lakatos's technique of lemma incorporation, may then be understood as a transformation on a Toulmin layout, as represented in Fig. 7. Here we would generally expect $Q^{\prime} \leq Q$, since removing the rebuttal should have strengthened the argument. It is also likely that $W^{\prime}=W$ and $B^{\prime}=B$, since the only additional support required for the new layout is a valid inference in most systems of $\operatorname{logic}(\sim L=L \Rightarrow C)$. With Lakatos's Rule 4 we see a genuine dividend in the new idiom: whereas Lakatos's focus is propositional, Toulmin's is on the process of argument. Lakatos's account of this move only permits the replacement of one proposition, 'the refuted lemma', with another; our model permits the replacement of a rebutted section of a proof network, including not just data, but also warrants, backing, qualifiers, and rebuttals. 


\section{Bibliography}

Aberdein, A.: The informal logic of mathematical proof. In R. Hersh, ed., $18 \mathrm{Un}$ conventional Essays about the Nature of Mathematics, Springer-Verlag, New York, NY, 2006.

Govier, T.: Problems in Argument Analysis and Evaluation, Foris, Dordrecht, 1987.

Johnson, R.: The Rise of Informal Logic, Vale, Newport News, VA, 1996.

Jones, V.: A credo of sorts. In H. G. Dales, G. Oliveri, eds., Truth in Mathematics, Clarendon, Oxford, 1998.

Kirschner, P. A., Buckingham Shum, S. J., Carr, C. S., eds.: Visualizing Argumentation: Software Tools for Collaborative and Educational Sense-Making, Springer-Verlag, London, 2003.

Kohlhase, M.: OMDoc: An open markup format for mathematical documents. Seki Report SR-00-02, Fachbereich Informatik, Universität des Saarlandes, 2000, www.mathweb.org/omdoc.

Lakatos, I.: Proofs and Refutations: The Logic of Mathematical Discovery, J. Worrall, E. Zahar, eds., Cambridge University Press, Cambridge, 1976.

Lamport, L.: How to write a proof. American Mathematical Monthly, 102(7) (1995) 600-608.

Littlewood, J. E.: Littlewood's Miscellany, B. Bollobás, ed., Cambridge University Press, Cambridge, 1986.

Newman, S., Marshall, C.: Pushing Toulmin too far: Learning from an argument representation scheme, Xerox PARC technical report no. SSL-92-45, 1992, www.csdl.tamu.edu/ marshall/toulmin.pdf.

Reed, C., Rowe, G.: Toulmin diagrams in theory \& practice: Theory neutrality in argument representation. In D. Hitchcock, D. Farr, eds., The Uses of Argument: Proceedings of a Conference at McMaster University, 18-21 May 2005, OSSA, Hamilton, ON, 2005.

Toulmin, S.: The Uses of Argument, Cambridge University Press, Cambridge, 1958.

Toulmin, S., Rieke, R., Janik, A.: An Introduction to Reasoning, Macmillan, New York, NY, 1979. 